\title{
Effects of $\mathrm{LaNiO}_{3}$ Seed Layer on the Microstructure and Electrical Properties of Ferroelectric BZT/PZT/ BZT Thin Films
}

\author{
Jinyu Ruan ${ }^{1}$, Chao Yin ${ }^{1,2}$, Tiandong Zhang ${ }^{1,2 *}$ and Hao Pan ${ }^{3 *}$ \\ ${ }^{1}$ School of Physics and Electronic Technology, Liaoning Normal University, Dalian, China, ${ }^{2}$ Key Laboratory of Engineering \\ Dielectrics and Its Application, Ministry of Education, Harbin, China, ${ }^{3}$ School of Physical and Mathematical Sciences, Nanyang \\ Technological University, Jurong West, Singapore
}

OPEN ACCESS

Edited by:

Weiwei $\mathrm{Li}$,

University of Cambridge,

United Kingdom

Reviewed by:

Renlu Han,

Ningbo University, China

Zhen Fan,

South China Normal University, China

Athipong Ngamjarurojana,

Chiang Mai University, Thailand

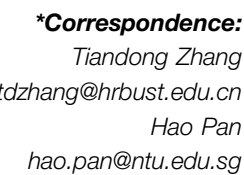

Specialty section:

This article was submitted to

Thin Solid Films,

a section of the journal

Frontiers in Materials

Received: 28 June 2021

Accepted: 03 August 2021 Published: 15 September 2021

Citation:

Ruan J, Yin C, Zhang T and Pan H (2021) Effects of $\mathrm{LaNiO}_{3}$ Seed Layer on

the Microstructure and Electrical

Properties of Ferroelectric BZT/PZT/

BZT Thin Films.

Front. Mater. 8:732186.

doi: 10.3389/fmats.2021.732186
Ferroelectric multilayer films attract great attention for a wide variation of applications. The synergistic effect by combining different functional layers induces distinctive electrical properties. In this study, ferroelectric $\mathrm{BaZr}_{0.2} \mathrm{Ti}_{0.8} \mathrm{O}_{3} / \mathrm{PbZr}_{0.52} \mathrm{Ti}_{0.48} \mathrm{O}_{3} /$ $\mathrm{BaZr}_{0.2} \mathrm{Ti}_{\mathrm{O}_{8.8} \mathrm{O}_{3}}$ (BZT/PZT/BZT) multilayer thin films are designed and fabricated by using the magnetron sputtering method, and a LaNiO3 (LNO) seed layer is introduced. The microstructures and electrical properties of the BZT/PZT/BZT films with and without the LNO seed layer are systematically studied. The results show that the BZT/PZT/BZT/LNO thin film exhibits much lower surface roughness and a preferred (100)-orientation growth, with the growth template and tensile stress provided by the LNO layer. Moreover, an enhanced dielectric constant, decreased dielectric loss, and improved ferroelectric properties are achieved in BZT/PZT/BZT/LNO thin films. This work reveals that the seed layer can play an important role in improving the microstructure and properties of ferroelectric multilayer films.

Keywords: magnetron sputtering, multilayer films, seed layer, microstructure, electrical property

\section{INTRODUCTION}

Ferroelectric thin films have been widely studied for several decades, which exhibit excellent dielectric, piezoelectric, and ferroelectric properties, which can be widely used as telecommunications, sensors, transducers, and so on. Representative ferroelectric materials such as $\mathrm{Pb}(\mathrm{Zr}, \mathrm{Ti}) \mathrm{O}_{3}$ (Damjanovic, 1998; Muralt, 2008; Palukurua et al., 2014), $\mathrm{Pb}\left(\mathrm{Mg}_{1 / 3} \mathrm{Nb}_{2 / 3}\right) \mathrm{O}_{3}-\mathrm{PbTiO}_{3}$ (Feng et al., 2011; Crossley et al., 2016), $\mathrm{Ba}(\mathrm{Zr}, \mathrm{Ti}) \mathrm{O}_{3}$ (Cao and Li, 2009; Fan et al., 2010; Luo et al., 2013), $\left(\mathrm{Bi}_{1 / 2} \mathrm{Na}_{1 / 2}\right) \mathrm{TiO}_{3}$ (Feng et al., 2016; Lin et al., 2016), and $\mathrm{BiFeO}_{3}$ (Rojacl et al., 2017; Campanini et al., 2018) have been intensively investigated and well-developed. With the increasing awareness of environmental protection, many studies strive to replace $\mathrm{Pb}$-based thin films with $\mathrm{Pb}$ free ones. Although progress has been made in $\mathrm{Pb}$-free films, the electrical properties in most cases are still inferior to those of $\mathrm{Pb}$-based ones.

Constructing multilayer thin films may be a compromise strategy to reduce the usage of lead and retain the outstanding characteristics of lead materials. Moreover, a novel physical phenomenon and exceptional properties may be achieved by combining the lead-free films with lead-based ones. For example, the $\mathrm{BiFeO}_{3} / \mathrm{PbZr}_{0.5} \mathrm{Ti}_{0.5} \mathrm{O}_{3}$ multilayer films exhibit not only reduced leakage current but also enhanced dielectric properties compared with pure 

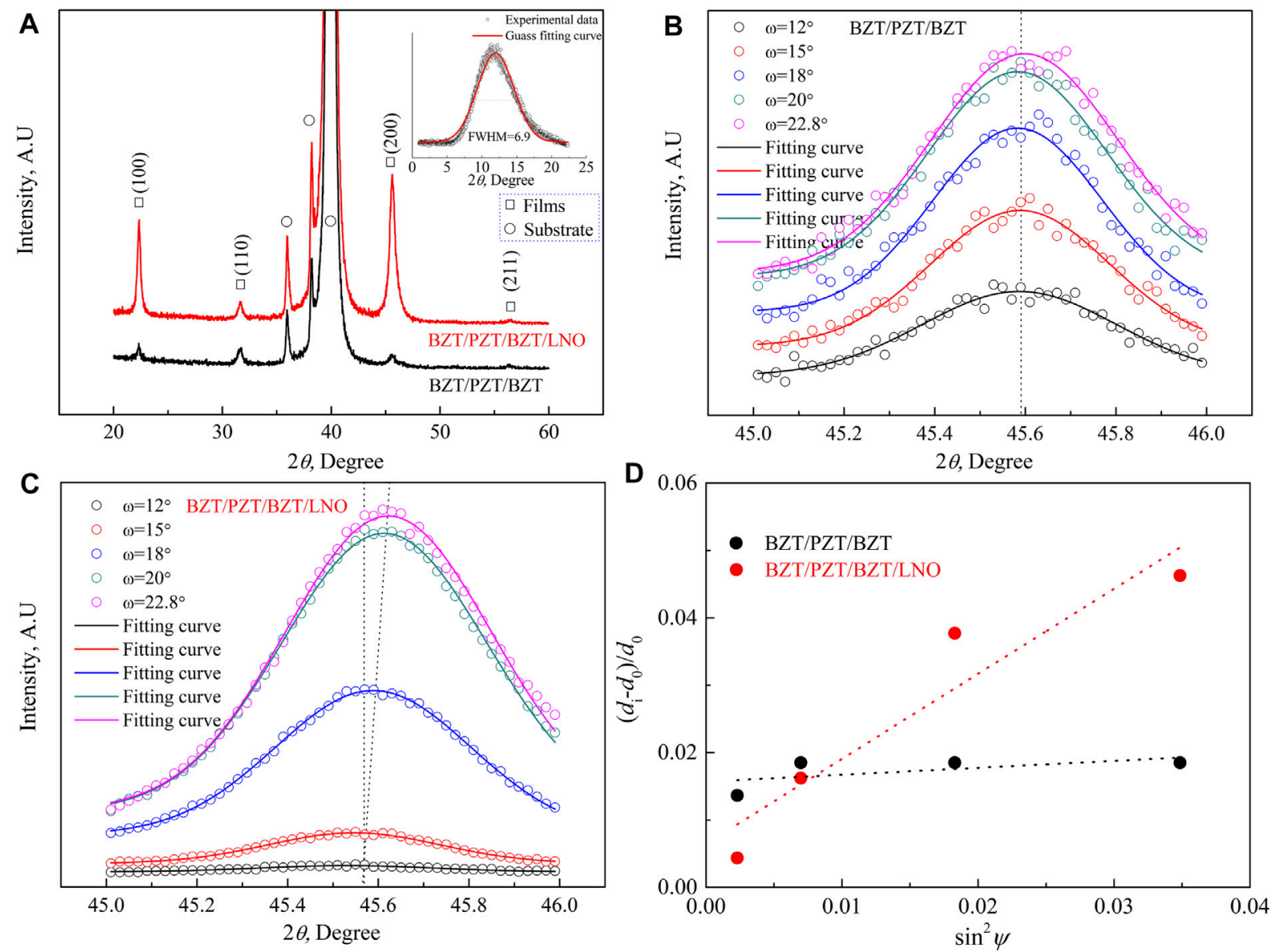

FIGURE 1 |XRD patterns of the BZT/PZT/BZT multilayer films. (A) $\theta-2 \theta$ scan patterns; the inset is the rocking curve patterns. (B, C) GIXD of (001) peaks of BZT/ PZT/BZT and BZT/PZT/BZT/LNO multilayer films. (D) Dependence of the interplanar crystal spacing on $\sin ^{2} \psi$.

$\mathrm{BiFeO}_{3}$ films (Li et al., 2005). Dielectric anomaly was also observed in $\mathrm{PbZr}_{0.2} \mathrm{Ti}_{0.8} \mathrm{O}_{3} / \mathrm{SrTiO}_{3}$ (ferroelectric/paraelectric) bilayer films due to the polarization mismatch. A maximum dielectric constant could be obtained when the layer thickness ratio reached a critical value (Khassaf et al., 2016). Besides, compositionally multilayer thin films (Damodaran et al., 2017a) and heterostructure thin films (Damodaran et al., 2017b; Zhang et al., 2017) also exhibit outstanding electrical properties. In addition, in bilayer films composed of a ferromagnetic NiFe or $\mathrm{CoFe}_{2} \mathrm{O}_{4}$ layer and ferroelectric $\mathrm{Pb}(\mathrm{Zr}, \mathrm{Ti}) \mathrm{O}_{3}$ layer, a remarkable magnetoelectric effect was obtained (Li et al., 2016; RamírezCamacho et al., 2017).

It is well-known that the growth orientations and electrical properties of ferroelectric thin films are very sensitive to the stress from the substrate, seed layer, buffer layer, or interfacial layer. For example, $\mathrm{BaZr}_{0.2} \mathrm{Ti}_{0.8} \mathrm{O}_{3}$ (BZT) films with a preferred (100) orientation can be obtained by using a $\mathrm{La}_{0.7} \mathrm{Sr}_{0.3} \mathrm{MnO}_{3}$ bottom layer (Tang et al., 2006) or $\mathrm{LaNiO}_{3}$ (LNO) seed layer (LiZhang et al., 2015) on $\mathrm{Pt} / \mathrm{Ti} / \mathrm{SiO}_{2} / \mathrm{Si}$ substrates in which the dielectric and piezoelectric properties were substantially improved. Specifically, LNO was widely used to regulate the microstructure and growth orientation (Li et al., 2010; Zhu et al., 2016) and to optimize the piezoelectric coefficient (LiZhang et al., 2014; LiZhang et al., 2015), polarization behavior (Hou et al., 2015), and photovoltaic current (Cheng, et al., 2020) of ferroelectric films. In this study, $\mathrm{BaZr}_{0.2} \mathrm{Ti}_{0.8} \mathrm{O}_{3} \quad(\mathrm{BZT}) / \mathrm{PbZr}_{0.52} \mathrm{Ti}_{0.48} \mathrm{O}_{3} \quad(\mathrm{PZT}) / \mathrm{BZT}$ multilayer thin films are constructed for performance enhancement, and the LNO is chosen as a seed layer for the film growth. The effects of LNO seed layer on the microstructure and electrical properties are systematically studied in this work.

\section{EXPERIMENTAL SECTION}

An LNO seed layer of $\sim 10 \mathrm{~nm}$ in thickness was grown on $\mathrm{Pt}$ $(111) / \mathrm{Ti} / \mathrm{SiO}_{2} / \mathrm{Si}$ substrates by a sol-gel method, as reported in 



FIGURE 2 | Surface morphology images of the BZT/PZT/BZT and BZT/PZT/BZT/LNO thin films. (A, B) SEM images; (C, D) AFM images.

our previous study (LiZhang et al., 2015). The raw materials were lanthanum nitrate $\left[\mathrm{La}\left(\mathrm{NO}_{3}\right)_{3}\right]$ and nickel acetate $\left[\mathrm{Ni}\left(\mathrm{CH}_{3} \mathrm{COO}\right)_{2}\right] . \quad \mathrm{La}\left(\mathrm{NO}_{3}\right)_{3}$ and $\mathrm{Ni}\left(\mathrm{CH}_{3} \mathrm{COO}\right)_{2}$ were dissolved and refluxed for $30 \mathrm{~min}$ in the heated 2methoxyethanol solvent, and the concentration of the solution was adjusted to $0.05 \mathrm{~mol} / \mathrm{L}$. After the precursor solution was aged for $24 \mathrm{~h}$, the LNO layer was spin-coated on the $\mathrm{Pt}(111) / \mathrm{Ti} / \mathrm{SiO}_{2} / \mathrm{Si}$ substrates, followed by pyrolysis at $400^{\circ} \mathrm{C}$ for $3 \mathrm{~min}$, and then annealing at $750^{\circ} \mathrm{C}$ for $30 \mathrm{~min}$. BZT and PZT thin films were then grown on the LNO/Pt (111)/Ti/ $\mathrm{SiO}_{2} / \mathrm{Si}$ substrates with a magnetron sputtering method. A base pressure of $5 \times 10^{-5} \mathrm{~Pa}$ was achieved before sputtering, and the Ar-to- $\mathrm{O}_{2}$ flow ratio was 3:1 during film growth. The top and bottom BZT layers are $\sim 150 \mathrm{~nm}$ in thickness, and the PZT interlayer is $\sim 200 \mathrm{~nm}$ in thickness. Finally, the prepared BZT/ $\mathrm{PZT} / \mathrm{BZT}$ and BZT/PZT/BZT/LNO thin films were annealed at $750^{\circ} \mathrm{C}$ for $30 \mathrm{~min}$ to enhance crystallization.

The crystalline structures of the thin films were analyzed by a Philips $\mathrm{X}^{\prime}$ pert X-ray diffractometer (XRD) with $\mathrm{Cu} \mathrm{K} \otimes$ radiation generated at $40 \mathrm{kV}$ and $40 \mathrm{~mA}$. The cross-section micrographs of the thin films were obtained by using scanning electron microscopy (SEM, Helios Nanolab600i), and the film surface morphology was characterized by an atomic force microscope (AFM). Piezoelectric response was detected by a piezoelectric force microscope (PFM). For further electrical property measurements, platinum electrodes with a diameter of $200 \mu \mathrm{m}$ were deposited by DC magnetron sputtering. The ferroelectric properties and leakage current were characterized by a Radiant Precision Workstation Ferroelectric Measurement System. The frequency and temperature dependent dielectric properties were measured on a precision impedance analyzer (Agilent 4294A), with an AC voltage of $500 \mathrm{mV}$.

\section{RESULTS AND DISCUSSION}

The XRD measurement shows a pure perovskite phase of the films (Figure 1A). It is found that the BZT/PZT/BZT/LNO thin films possess preferred (100)-oriented growth, while the BZT/ PZT/BZT films without the LNO seed layer do not exhibit an obvious crystal orientation preference. The XRD rocking curve $(\omega$ 

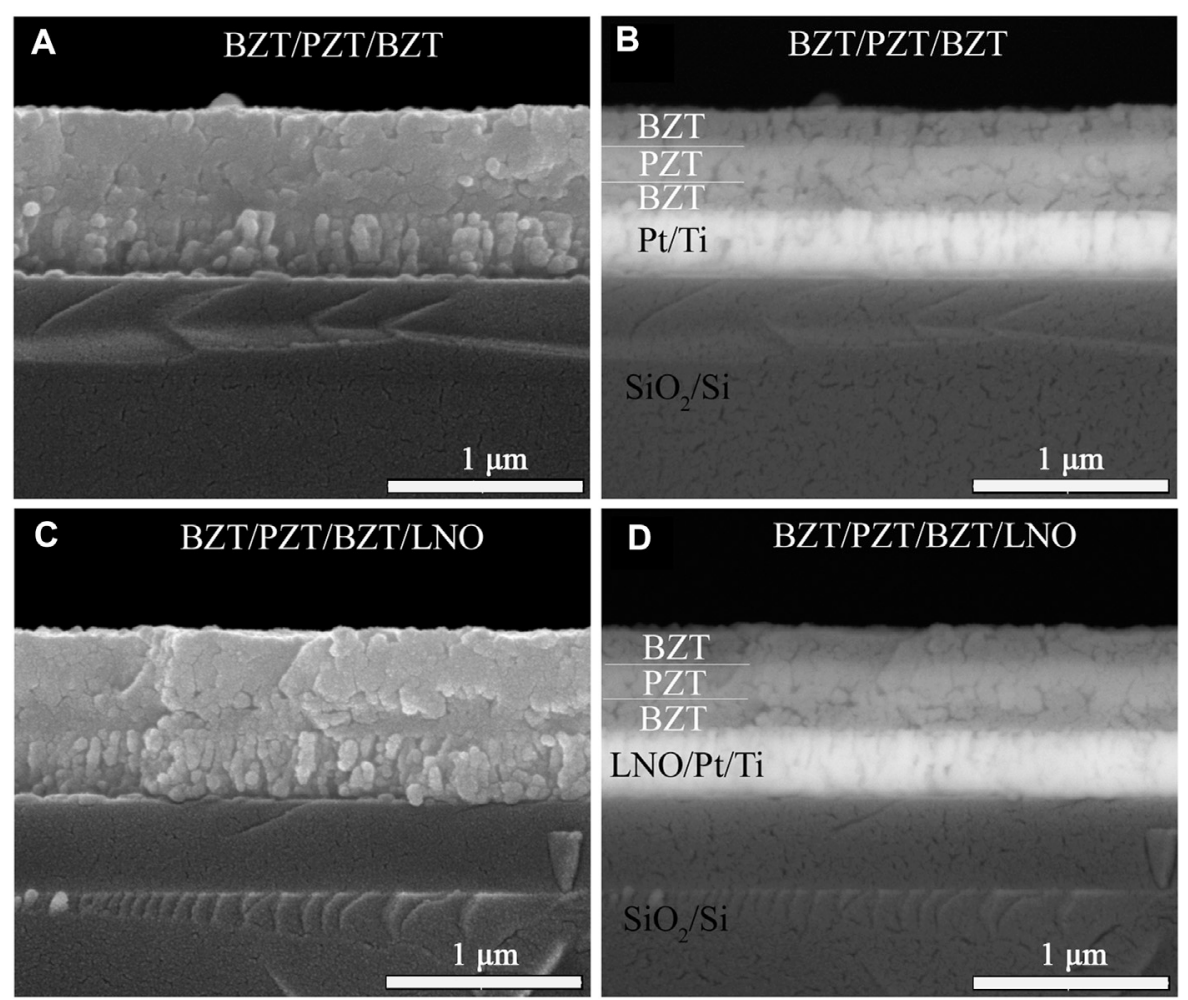

FIGURE 3 | Cross-sectional images of the BZT/PZT/BZT and BZT/PZT/BZT/LNO thin films. (A, C) SEM images; (B, D) BSE images.

scan) of the BZT/PZT/BZT/LNO film exhibits a full width at a half maximum (FWHM) of $6.9^{\circ}$ (the inset of Figure 1A). This small FWHM value reflects the strong orientation preference induced by the LNO seed layer. There are no diffraction peaks of the LNO seed layer due to its small thickness, consistent with our previous studies (LiZhang et al., 2015). To study the stress state in the $\mathrm{BZT} / \mathrm{PZT} / \mathrm{BZT}$ thin films, the grazing incidence $\mathrm{X}$-ray diffraction (GIXD) method (Hou et al., 2015) was employed, and the results are shown in Figures 1B,C. According to the equation of $\psi=\theta-\omega$ ( $\theta$ is incident angle, $\omega$ is the grazing incident angle, and $\psi$ is the included angle between the normal direction of films and diffraction crystal plane), $\psi$ can be obtained by changing the $\omega$ value. Furthermore, the slightly changed $\theta$ value means the variation of the interplanar crystal spacing $d_{\mathrm{i}}$, which can be calculated by the Bragg's equation $2 d_{\mathrm{i}} \sin \theta=\lambda$, [here, $d_{0}$ is the interpalne spacing of stress-free specimen and $\left(d_{\mathrm{i}}-d_{0}\right) / d_{0}$ corresponds the crystal lattice strain]. It should be noted that there is a linear relationship between the $\left(d_{\mathrm{i}}-d_{0}\right) / d_{0}$ and $\sin ^{2} \psi$. (For detailed derivation process, refer the previous reports (Wang et al., 2010).) The increased incident angle $\omega$ corresponds to the increasing penetration depth of X-ray. Therefore, the strain distribution behavior along the normal direction in the films can be revealed by the GIXD method with different $\omega$ values. In this work, the GIXD peaks of the (200) planes are shown in
Figures 1B,C, respectively, for the BZT/PZT/BZT and BZT/PZT/ $\mathrm{BZT} / \mathrm{LNO}$ thin films. It can be seen that the diffraction peak is almost unchanged for the BZT/PZT/BZT films, which, as a comparison, exhibits an increased two theta with the increasing $\omega$ in the $\mathrm{BZT} / \mathrm{PZT} / \mathrm{BZT} / \mathrm{LNO}$ films. It means that the LNO seed layer induces tensile stress in the BZT/PZT/BZT thin films, leading to an increased interplanar spacing $d_{\mathrm{i}}$, as shown in Figure 1D.

Figure 2 shows the surface morphology of the films. The films fabricated by magnetron sputtering exhibit island growth characteristics. SEM images show that the grain size is about $1 \mu \mathrm{m}$, with some fine grains of $\sim 100 \mathrm{~nm}$ distributed sporadically between the adjacent large grains (Figures $\mathbf{2 A}, \mathbf{B})$. In order to obtain more detailed surface information, AFM characterization was employed, and the results are given in Figures 2C,D. It can be clearly found that a more compact and smoother surface structure is obtained in the $\mathrm{BZT} / \mathrm{PZT} / \mathrm{BZT} / \mathrm{LNO}$ films. The film roughness was evaluated by the root-mean-square (RMS) values derived from the AFM measurement, which are 16.7 and $7.4 \mathrm{~nm}$ for the $\mathrm{BZT} / \mathrm{PZT} / \mathrm{BZT}$ and $\mathrm{BZT} / \mathrm{PZT} / \mathrm{BZT} / \mathrm{LNO}$ thin films, respectively. The results indicate that the LNO seed layer is beneficial to improve the film quality and to reduce the surface roughness. 

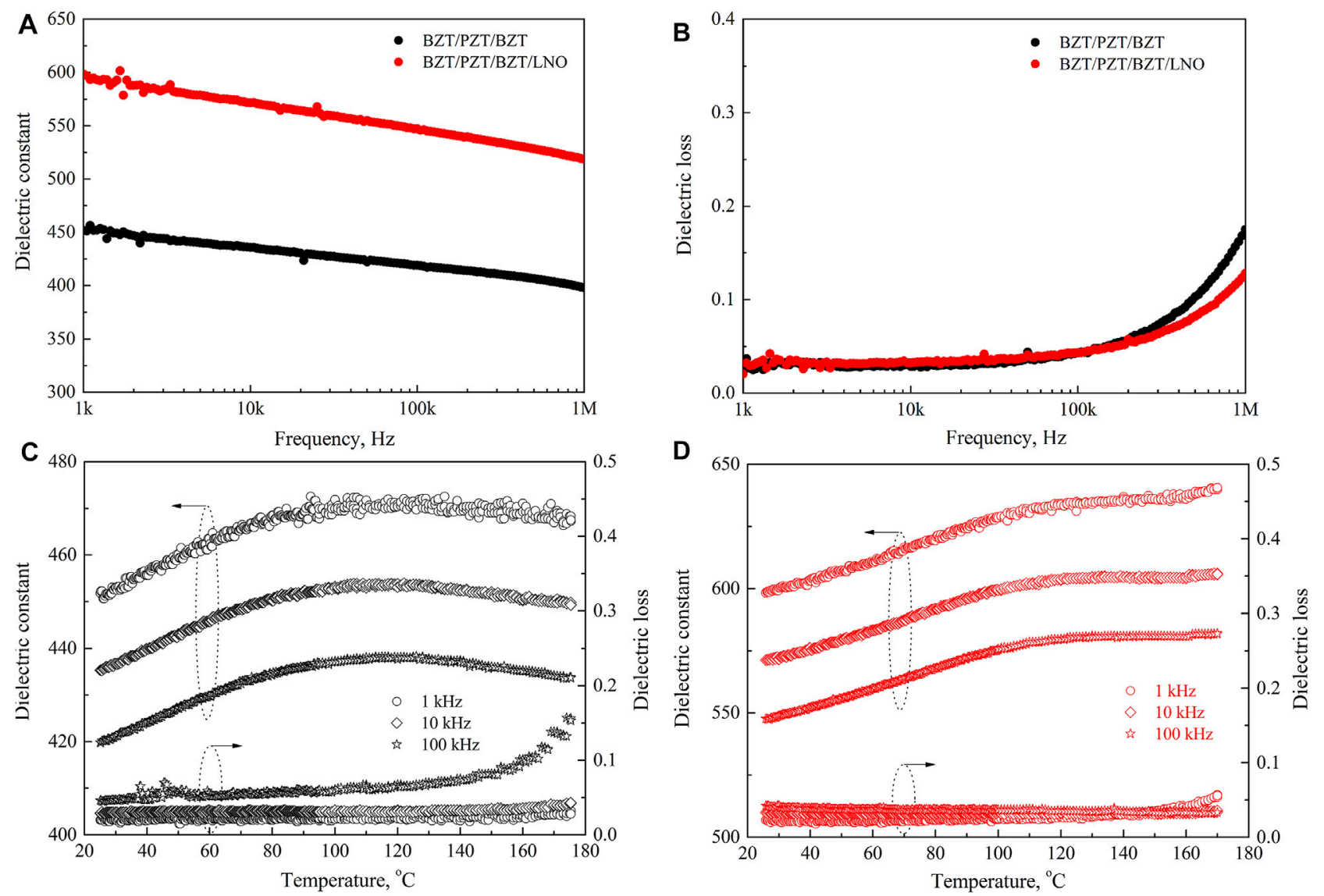

FIGURE 4 | Dependence of dielectric properties on measured frequency and temperature. (A) Dielectric constant. (B) Dielectric loss. (C) BZT/PZT/BZT. (D) BZT/ PZT/BZT/LNO.

Cross-sectional SEM images of the films are shown in Figure 3. It can be seen that the $\mathrm{BZT} / \mathrm{PZT} / \mathrm{BZT} / \mathrm{LNO}$ thin film possesses a much denser microstructure than that of the BZT/PZT/BZT thin film without the LNO seed layer. Some voids between adjacent grains can be seen in the BZT/PZT/BZT thin film, which is much more obviously seen in the backscattered electron (BSE) images, as given in Figures 3B,D. The total thickness of the films is verified to be $\sim 500 \mathrm{~nm}$, with the thickness of the top and bottom BZT layers of about $150 \mathrm{~nm}$, and the PZT interlayer of about $200 \mathrm{~nm}$. The interface between BZT and PZT can be clearly observed due to the different atomic numbers of $\mathrm{Ba}$ and $\mathrm{Pb}$, and thus the varied contrast in the BSE image. The LNO seed layer is too thin to observe from the SEM images, whose thickness was estimated to be $\sim 10 \mathrm{~nm}$ via the X-ray reflectivity method (LiZhang et al., 2015).

Since dielectric properties are very important for ferroelectric thin films, the dependence of dielectric properties on the measurement frequency and temperature is investigated, and the results are given in Figure 4. It can be found that the dielectric constant is notably improved by introducing an LNO seed layer into the BZT/PZT/BZT film. The dielectric constants are 451.7 and 598.6, respectively, for the BZT/PZT/BZT and BZT/PZT/BZT/LNO thin films at $1 \mathrm{kHz}$. As shown in Figure $4 \mathrm{~B}$, the dielectric loss of the $\mathrm{BZT} / \mathrm{PZT} / \mathrm{BZT} / \mathrm{LNO}$ thin film at higher frequencies is lower than that of BZT/PZT/BZT/LNO thin films. The enhanced dielectric constant and the decreased dielectric loss can be ascribed to the improved film quality and polarization capability by inserting the LNO seed layer, which is consistent with the results of previous reports ( $\mathrm{Wu}$ and Shy, 2000; Gao et al., 2008). The temperature-dependent dielectric properties of the films are presented in Figures 4C,D. It can be observed that both the BZT/PZT/BZT and the BZT/PZT/BZT/ LNO thin films possess a wide peak of dielectric constant, which may correspond to the transition from the rhombohedral phase to the cubic phase of BZT (Lee et al., 2005; Xu et al., 2015). Such broad peaks could also link to the 

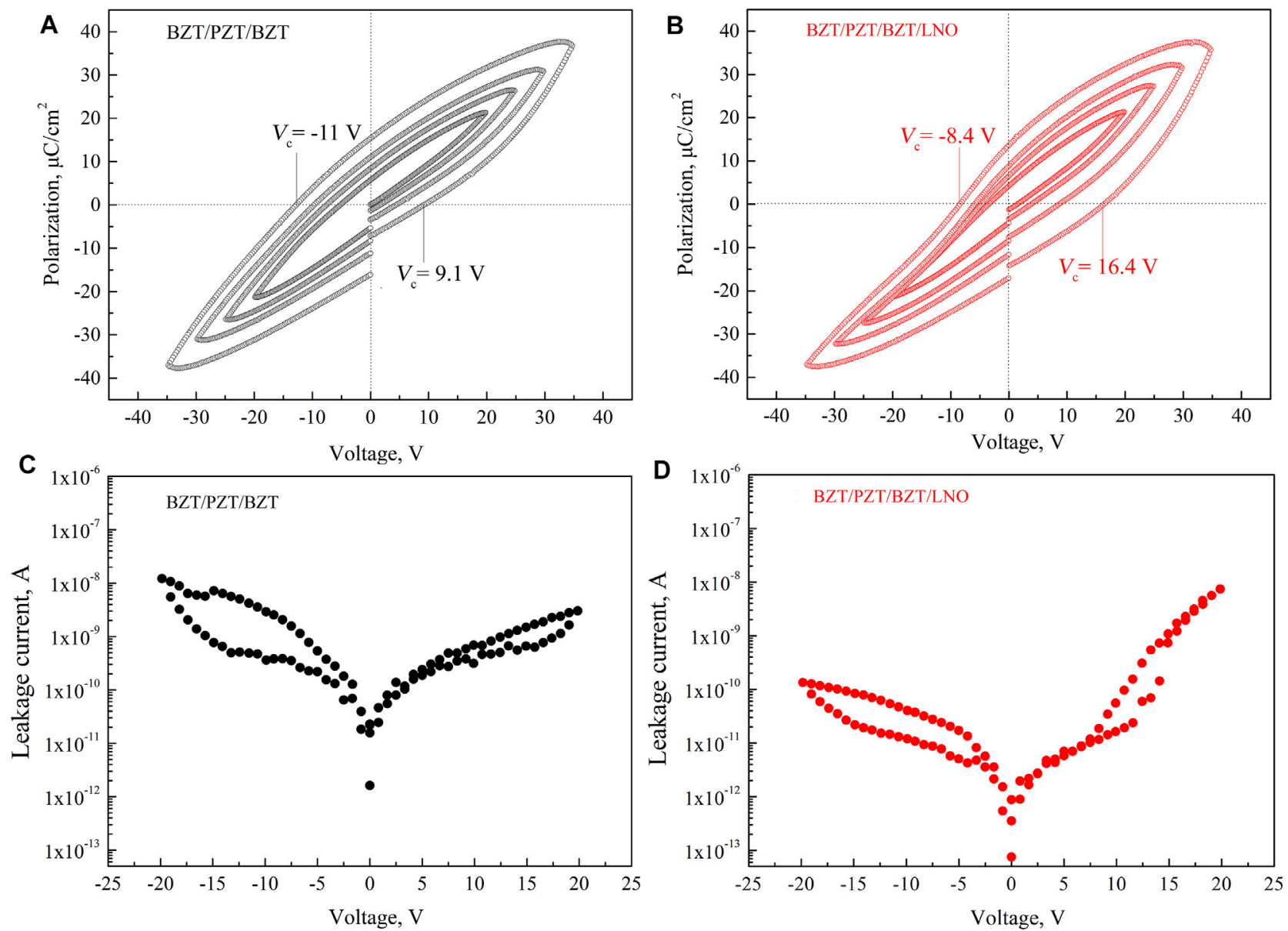

FIGURE 5 | Hysteresis loops and leakage current with different applied voltages. (A, C) BZT/PZT/BZT thin films. (B, D) BZT/PZT/BZT/LNO thin films.

diffuse phase transitions due to the substrate clamping effect (Setter, et al., 2006; Tong, et al., 2011). Besides, the BZT/PZT/ $\mathrm{BZT} / \mathrm{LNO}$ film maintains lower and more stable dielectric loss values as the temperature increases, which is also associated with the higher quality and less defective microstructure of the film with the LNO seed layer.

Ferroelectric properties of the films are investigated by measuring hysteresis polarization-voltage loops. As given in Figure 5A, the hysteresis loops of the BZT/PZT/BZT thin film are not completely closed, and an obvious gap can be seen at the negative branch as the applied voltage decreases to zero. This phenomenon may be correlated with the leakage current or conduction loss, which further leads to the asymmetric coercive voltage $\left(V_{c}\right)$ values. As a comparison, it can be noticed that the hysteresis loops of the BZT/PZT/BZT/LNO film is almost closed after the voltage cycle, indicating a lower leakage current. It should be noted that the obviously asymmetric coercive voltage values still exist in the BZT/PZT/BZT/LNO thin film, with the $+V_{c}$ of $16.4 \mathrm{~V}$ and $-V_{c}$ of $-8.4 \mathrm{~V}$. It was reported that the loop offset along the voltage axis can be induced by the asymmetric electrode structure of thin films (Lee et al., 2005; Wong and Shin, 2005). In addition, the interfacial charge in the multilayer films and the existence of built-in electric field may also lead to the asymmetrical characteristics of hysteresis loops (Karthik et al., 2013; Agar et al., 2015).

Figures 5C,D show the leakage current of the thin films at different voltages. The leakage current of the BZT/PZT/BZT/ LNO film is generally lower than that of the BZT/PZT/BZT film. Besides, in the BZT/PZT/BZT/LNO thin film, the leakage current exhibits an unsymmetrical characteristic, which is higher at positive bias voltages. A possible reason may be the asymmetric electrodes that lead to different interfacial Schottky barrier heights and induce the unsymmetrical characteristics of $I V$ curve (Shen et al., 2014; Fan et al., 2015; Chen et al., 2020). 

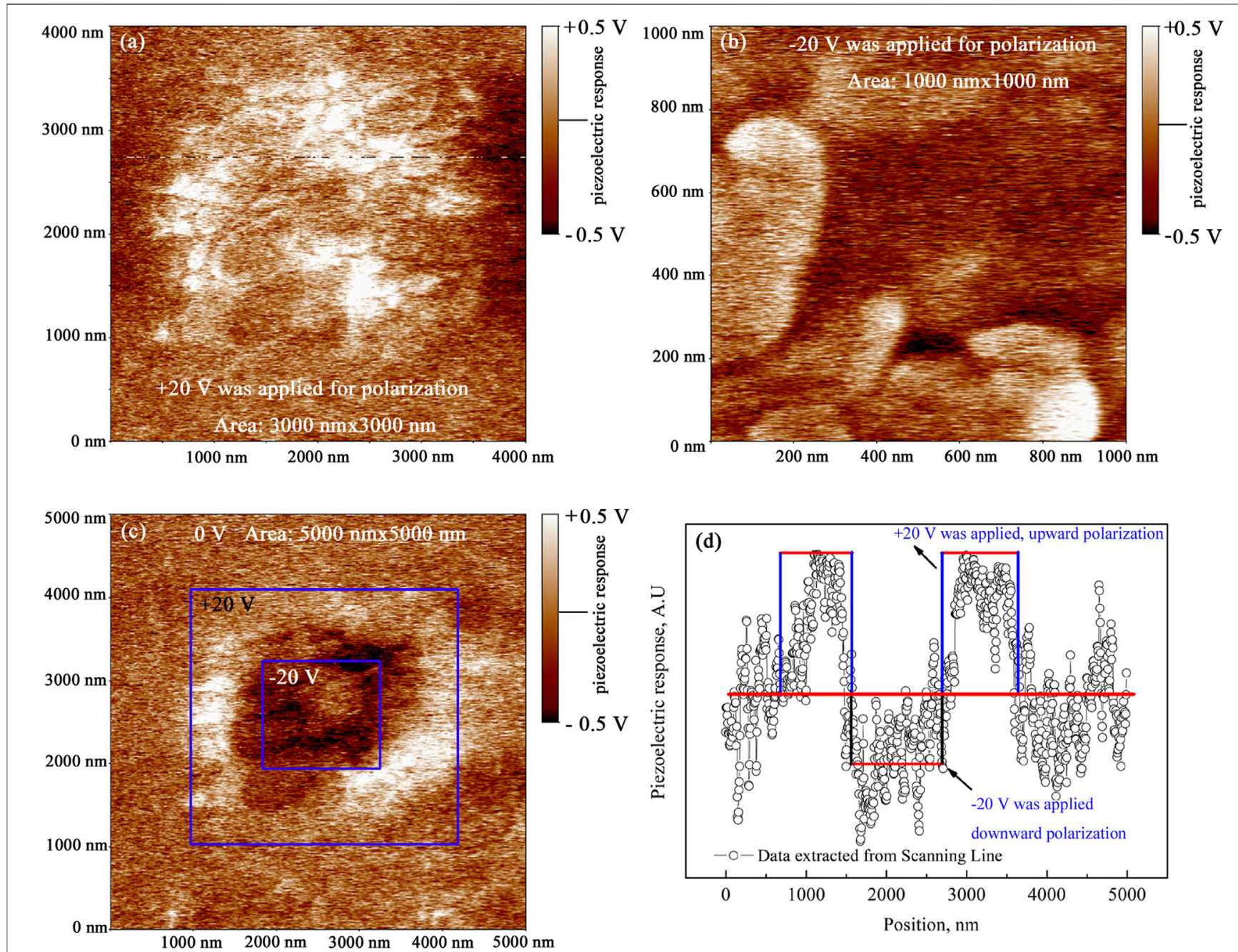

FIGURE 6 | PFM images of the BZT/PZT/BZT/LNO thin film. (A) + $20 \mathrm{~V}$ polarization voltage. (B) $-20 \mathrm{~V}$ polarization voltage. (C) Scanning after voltage was removed. (D) Piezoelectric response extracted from a scanning line in Fig. (C).

To further study the effects of the LNO seed layer on the polarization behavior of thin films, piezoelectric force microscopy (PFM) was employed to directly observe the polarization reversal behavior, based on the evolution of piezoelectric response. A positive voltage and a negative voltage were applied sequentially on the BZT/PZT/BZT/LNO film. A 3,000-nm $\times 3,000-\mathrm{nm}$ area at the center of the selected region was first poled by $+20 \mathrm{~V}$ voltage (Figure 6A), and then a 1,000-nm $\times 1,000-\mathrm{nm}$ area at the center was poled by an inversed $-20 \mathrm{~V}$ voltage to study the polarization reversal (Figure 6B). It should be pointed out that the voltage was applied between the bottom electrode and PFM tip, and the tip was grounded. Finally, the PFM signals of the $5,000 \mathrm{~nm} \times$ $5,000 \mathrm{~nm}$ area were scanned after removing the voltage (Figure 6C). Because the piezoelectric response signal is proportional to the out-of-plane polarization of the film, the bright regions correspond to upward polarization, and dark regions to downward polarization. In Figure 6D, one can find that the intensity of the positive piezoelectric response is stronger than that of the negative piezoelectric response, meaning that thin films are easier to be polarized when a positive polarization voltage is applied.

\section{CONCLUSIONS}

In this study, BZT/PZT/BZT/LNO thin films were fabricated by combining magnetron sputtering and sol-gel methods. The results show that the (100) preferred orientation growth of the BZT/PZT/ BZT thin film is induced by introducing an LNO seed layer, with which a denser microstructure and lower surface roughness are achieved. More importantly, the dielectric constant increases and the dielectric loss decreases with the insertion of the LNO layer, accompanied by a diffuse phase transition behavior. Besides, asymmetric hysteresis loops and leakage current curves are found in the BZT/PZT/BZT/LNO thin film, which can be attributed to the LNO seed layer that may cause different polarization abilities under 
different electric field directions. The PFM measurement also supports the above deduction. This work provides an effective way to improve the microstructure and electrical properties of ferroelectric multilayer thin films by introducing an LNO seed layer.

\section{DATA AVAILABILITY STATEMENT}

The original contributions presented in the study are included in the article/Supplementary Material; further inquiries can be directed to the corresponding authors.

\section{REFERENCES}

Agar, J. C., Damodaran, A. R., Velarde, G. A., Pandya, S., Mangalam, R. V. K., and Martin, L. W. (2015). Complex Evolution of Built-in Potential in Compositionally-Graded PbZr1-xTixO3 Thin Films. ACS Nano 9, 7332-7342. doi:10.1021/acsnano.5b02289

Campanini, M., Erni, R., Yang, C. H., Ramesh, R., and Rossell, M. D. (2018). Periodic Giant Polarization Gradients in Doped BiFeO3 Thin Films. Nano Lett. 18, 717-724. doi:10.1021/acs.nanolett.7b03817

Cao, H.-X., and Li, Z.-Y. (2009). Electrocaloric effect in BaTiO3 thin films. J. Appl. Phys. 106, 094104. doi:10.1063/1.3253736

Chen, A., Zhang, W., Dedon, L. R., Chen, D., Khatkhatay, F., MacManus-Driscoll, J. L., et al. (2020). Couplings of polarization with interfacial deep trap and schottky interface controlled ferroelectric memristive switching. Adv. Funct. Mater. 30, 2000664. doi:10.1002/adfm.202000664

Cheng, S., Fan, Z., Rao, J., Hong, L., Huang, Q., Tao, R., et al. (2020). Highly controllable and silicon-compatible ferroelectric photovoltaic synapses for neuromorphic computing. iScience 23, 101874. doi:10.1016/ j.isci.2020.101874

Crossley, S., Usui, T., Nair, B., Kar-Narayan, S., Moya, X., Hirose, S., et al. (2016). Direct electrocaloric measurement of $0.9 \mathrm{~Pb}(\mathrm{Mg} 1 / 3 \mathrm{Nb} 2 / 3) \mathrm{O} 3-0.1 \mathrm{PbTiO} 3$ films using scanning thermal microscopy. Appl. Phys. Lett. 108, 032902. doi:10.1063/ 1.4938758

Damjanovic, D. (1998). Ferroelectric, dielectric and piezoelectric properties of ferroelectric thin films and ceramics. Rep. Prog. Phys. 61, 1267-1324. doi:10.1088/0034-4885/61/9/002

Damodaran, A. R., Clarkson, J. D., Hong, Z., Liu, H., Yadav, A. K., Nelson, C. T., et al. (2017). Phase coexistence and electric-field control of toroidal order in oxide superlattices. Nat. Mater 16, 1003-1009. doi:10.1038/NMAT4951

Damodaran, A. R., Pandya, S., Qi, Y., Hsu, S.-L., Liu, S., Nelson, C., et al. (2017). Large polarization gradients and temperature-stable responses in compositionally-graded ferroelectrics. Nat. Commun. 8, 14961. doi:10.1038/ ncomms14961

Fan, Y., Yu, S., Sun, R., Li, L., Yin, Y., and Du, R. (2010). Microstructure and electrical properties of $\mathrm{Ba} 0.7 \mathrm{Sr} 0.3(\mathrm{Ti} 1-\mathrm{xZrx}) \mathrm{O} 3$ thin films prepared on copper foils with sol-gel method. Thin Solid Films 518, 3610-3614. doi:10.1016/ j.tsf.2009.09.051

Fan, Z., Xiao, J., Yao, K., Zeng, K., and Wang, J. (2015). Ferroelectric polarization relaxation in $\mathrm{Au} / \mathrm{Cu} 2 \mathrm{O} / \mathrm{ZnO} / \mathrm{BiFeO} 3 / \mathrm{Pt}$ heterostructure. Appl. Phys. Lett. 106, 102902. doi:10.1063/1.4914883

Feng, C., Yang, C. H., Geng, F. J., Lv, P. P., and Yao, Q. (2016). Microstructure and electrical properties mediated by defects in $\mathrm{Na} 0.5 \mathrm{Bi} 0.5 \mathrm{Ti} 0.98 \mathrm{Mn} 0.02 \mathrm{O} 3$ thin film under different annealing atmospheres. J. Eur. Ceram. Soc. 36, 527-532. doi:10.1016/j.jeurceramsoc.2015.10.015

Feng, Z., Shi, D., Zeng, R., and Dou, S. (2011). Large electrocaloric effect of highly (100)-oriented $0.68 \mathrm{PbMg} 1 / 3 \mathrm{Nb} 2 / 3 \mathrm{O} 3-0.32 \mathrm{PbTiO} 3$ thin films with a $\mathrm{Pb}(\mathrm{Zr0}$.3Ti0.7)O3/PbOx buffer layer. Thin Solid Films 519, 5433-5436. doi:10.1016/j.tsf.2011.02.069

Gao, L. N., Song, S. N., Zhai, J. W., Yao, X., and Xu, Z. K. (2008). Effects of buffer layers on the orientation and dielectric properties of $\mathrm{Ba}(\mathrm{Zr} 0.20 \mathrm{Ti} 0.80) \mathrm{O} 3$ thin films prepared by sol-gel method. J. Cryst. Growth 310, 1245-1249. doi:10.1016/ j.jcrysgro.2007.12.015

\section{AUTHOR CONTRIBUTIONS}

JR: writing and investigation. CY: material synthesis. TZ: investigation and conceptualization. HP: writing and conceptualization.

\section{FUNDING}

The authors gratefully acknowledge support from the Outstanding Youth Fund of Heilongiiang Province (No. YQ2020E031). China Postdoctoral Science Foundation (No.2021T140166)

Hou, Y. F., Zhang, T. D., Li, W. L., Cao, W. P., Yu, Y., Xu, D., et al. (2015). Selfpolarization induced by lattice mismatch and defect dipole alignment in (001) $\mathrm{BaTiO} 3 / \mathrm{LaNiO} 3$ polycrystalline film prepared by magnetron sputtering at low temperature. RSC Adv. 5, 61821-61827. doi:10.1039/c5ra07337e

Karthik, J., Mangalam, R. V. K., Agar, J. C., and Martin, L. W. (2013). Large built-in electric fields due to flexoelectricity in compositionally graded ferroelectric thin films. Phys. Rev. B 87, 024111. doi:10.1103/PhysRevB.87.024111

Khassaf, H., Khakpash, N., Vijayan, S., Aindow, M., and Alpay, S. P. (2016). Electrostatically driven dielectric anomaly in mesoscopic ferroelectricparaelectric bilayers. Acta Materialia 105, 68-74. doi:10.1016/ j.actamat.2015.12.023

Lee, H. N., Christen, H. M., Chisholm, M. F., Rouleau, C. M., and Lowndes, D. H. (2005). Strong polarization enhancement in asymmetric three-component ferroelectric superlattices. Nature 433, 395-399. doi:10.1038/ nature0324810.1038/nature03261

Li, Y.-C., Pan, D.-F., WuLi, J. Y.-B., Li, Y.-b., Wang, G.-h., Liu, J.-M., et al. (2016). Manipulating the exchange bias effect of $\mathrm{Pb}(\mathrm{Zr} 0.52 \mathrm{Ti} 0.48) \mathrm{O} 3 / \mathrm{CoFe} 2 \mathrm{O} 4 / \mathrm{NiO}$ heterostructural films by electric fields. Appl. Phys. Lett. 109, 172904. doi:10.1063/1.4966545

Li, Y. W., Shen, Y. D., Yue, F. Y., Hu, Z. G., Ma, X. M., Chu, J. H., et al. (2010). Preparation and characterization of $\mathrm{BiFeO} 3 / \mathrm{LaNiO} 3$ heterostructure films grown on silicon substrate. J. Cryst. Growth 312, 617-620. doi:10.1016/ j.jcrysgro.2009.11.058

Li, Y. W., Sun, J. L., Chen, J., Meng, X. J., and Chu, J. H. (2005). Structural, ferroelectric, dielectric, and magnetic properties of $\mathrm{BiFeO} 3 \mathrm{~Pb}(\mathrm{Zr} 0.5, \mathrm{Ti} 0.5) \mathrm{O} 3$ multilayer films derived by chemical solution deposition. Appl. Phys. Lett. 87, 182902. doi:10.1063/1.2120907

Lin, Q., Ding, R., Li, Q., Tay, Y. Y., Wang, D., Liu, Y., et al. (2016). Large Piezoelectricity and Ferroelectricity in Mn-Doped (Bi $0.5 \mathrm{Na} 0.5$ ) TiO 3 BaTiO 3 Thin Film Prepared by Pulsed Laser Deposition. J. Am. Ceram. Soc. 99, 2347-2353. doi:10.1111/jace.14227

LiZhang, W. L. T. D., Zhang, T. D., Hou, Y. F., Zhao, Y., Xu, D., Cao, W. P., et al. (2014). Giant piezoelectric properties of BZT-0.5BCT thin films induced by nanodomain structure. RSC Adv. 4, 56933-56937. doi: $10.1039 / \mathrm{c} 4 \mathrm{ra} 08280 \mathrm{j}$

LiZhang, W. L. T. D., Zhang, T. D., Xu, D., Hou, Y. F., Cao, W. P., and Fei, W. D. (2015). LaNiO3 seed layer induced enhancement of piezoelectric properties in (100)-oriented (1-x)BZT-xBCT thin films. J. Eur. Ceram. Soc. 35, 2041-2049. doi:10.1016/j.jeurceramsoc

Luo, B. C., Wang, D. Y., Duan, M. M., and Li, S. (2013). Orientation-dependent piezoelectric properties in lead-free epitaxial 0.5BaZr0.2Ti0.8O30.5Ba0.7Ca0.3TiO3 thin films. Appl. Phys. Lett. 103, 122903. doi:10.1063/ 1.4821918

Muralt, P. (2008). Recent progress in materials issues for piezoelectric MEMS. J. Am. Ceram. Soc. 91, 1385-1396. doi:10.1088/0034-4885/61/9/00210.1111/ j.1551-2916.2008.02421.x

Palukuru, V. K., Lappalainen, J., Puustinen, J., Kangaspuoskari, M., and Huotari, J. (2014). Dielectric and Tunability Properties of Nanostructured Nd-modified $\mathrm{Pb}($ ZrxTi1-x)O3Thin Films up to Microwave Frequencies. Ferroelectrics 465 20-27. doi:10.1080/00150193.2014.893735

Ramírez-Camacho, M. C., Sánchez-Valdés, C. F., Gervacio-Arciniega, J. J., Font, R., Ostos, C., Bueno-Baques, D., et al. (2017). Room temperature ferromagnetism and ferroelectricity in strained multiferroic $\mathrm{BiFeO} 3$ thin films on 
$\mathrm{La} 0.7 \mathrm{Sr} 0.3 \mathrm{MnO} 3 / \mathrm{SiO} 2 / \mathrm{Si}$ substrates. Acta Materialia 128, 451-464. doi:10.1063/1.2120907

Rojac, T., Bencan, A., Drazic, G., Sakamoto, N., Ursic, H., Jancar, B., et al. (2017). Domain-wall conduction in ferroelectric $\mathrm{BiFeO} 3$ controlled by accumulation of charged defects. Nat. Mater 16, 322-327. doi:10.1038/ NMAT4799

Setter, N., Damjanovic, D., Eng, L., Fox, G., Gevorgian, S., Hong, S., et al. (2006). Ferroelectric thin films: Review of materials, properties, and applications. J. Appl. Phys. 100, 051606. doi:10.1063/1.2336999

Shen, L. M., Tang, X. G., Liu, Q. X., Jiang, Y. P., Wang, Y. G., and Li, W. P. (2014). Room temperature ferroelectric properties and leakage current characterics of $\mathrm{Bi} 2 \mathrm{FeMnO6} / \mathrm{SrTiO} 3$ bilayered thin films by chemical solution deposition. Phys. Status Solidi A. 211, 1499-1502. doi:10.1002/ pssa.201330617

Tang, X. G., Liu, Q. X., Jiang, Y. P., Zheng, R. K., and Chan, H. L. W. (2006). Enhanced dielectric properties of highly (100)-oriented $\mathrm{Ba}(\mathrm{Zr}, \mathrm{Ti}) \mathrm{O}$ [sub 3] thin films grown on $\mathrm{La}$ [sub 0.7] $\mathrm{Sr}$ [sub 0.3] $\mathrm{MnO}$ [sub 3] bottom layer. J. Appl. Phys. 100, 114105. doi:10.1063/1.2393010

Tong, S., Narayanan, M., Ma, B., Koritala, R. E., Liu, S., Balachandran, U., et al. (2011). Effect of dead layer and strain on the diffuse phase transition of PLZT relaxor thin films. Acta Materialia 59, 1309-1316. doi:10.1016/j.actamat.2010.10.063

Wang, J. N., Li, W. L., Feng, B., Liu, C. Q., Li, X. L., Sun, Q., et al. (2010). Residual stress of $\mathrm{Pb}($ ZrxTi1-x)O3 films with mixed textures. J. Alloys Comp. 506, 167-171. doi:10.1016/j.jallcom.2010.06.170

Wong, C. K., and Shin, F. G. (2005). A possible mechanism of anomalous shift and asymmetric hysteresis behavior of ferroelectric thin films. Appl. Phys. Lett. 86, 042901. doi:10.1063/1.1853520

Wu, T.-B., and Shy, H.-J. (2000). Deposition and properties of highly (100)oriented barium titanate thin films on $\mathrm{LaNiO} 3$ electrode. Ceramics Int. 26, 599-603. doi:10.1016/S0272-8842(99)00103-0
Xu, X., Yuan, G., Xu, B., Yin, J., Liu, J., and Liu, Z. (2015). Influence of the Strain on Dielectric and Ferroelectric Properties of $0.5 \mathrm{BaZr} 0.2$ Ti0.8 O3 -0.5Ba0.7 Ca0.3 TiO3. J. Am. Ceram. Soc. 98, 2823-2828. doi:10.1111/ jace. 13677

Zhang, Y.-J., Chen, J.-H., Li, L.-L., Ma, J., Nan, C.-W., and Lin, Y.-H. (2017). Ferroelectric strain modulation of antiferromagnetic moments in $\mathrm{Ni} / \mathrm{NiO}$ ferromagnet/antiferromagnet heterostructures. Phys. Rev. B 95, 174420. doi:10.1103/PhysRevB.95.174420

Zhu, M., Dong, X., Chen, Y., Xue, F., Lian, J., Xiao, L., et al. (2016). Growth control of RF magnetron sputtered SrRuO3 thin films through the thickness of $\mathrm{LaNiO}_{3}$ seed layers. Ceramics Int. 42, 13925-13931. doi:10.1016/ j.ceramint.2016.05.204

Conflict of Interest: The authors declare that the research was conducted in the absence of any commercial or financial relationships that could be construed as a potential conflict of interest.

Publisher's Note: All claims expressed in this article are solely those of the authors and do not necessarily represent those of their affiliated organizations, or those of the publisher, the editors, and the reviewers. Any product that may be evaluated in this article, or claim that may be made by its manufacturer, is not guaranteed or endorsed by the publisher.

Copyright (c) 2021 Ruan, Yin, Zhang and Pan. This is an open-access article distributed under the terms of the Creative Commons Attribution License (CC $B Y)$. The use, distribution or reproduction in other forums is permitted, provided the original author(s) and the copyright owner(s) are credited and that the original publication in this journal is cited, in accordance with accepted academic practice. No use, distribution or reproduction is permitted which does not comply with these terms. 